\title{
A Wearable Haptic System for the Health Monitoring of Elderly People in Smart Cities
}

\author{
https://doi.org/10.3991/ijoe.v14i08.8571 \\ Filippo Sanfilippo( $\left({ }^{\square}\right)$ \\ University College of Southeast Norway (USN), Kongsberg, Norway \\ filippo.sanfilippo@usn.no \\ Claudio Pacchierotti \\ University of Rennes, Rennes, France
}

\begin{abstract}
A sensor-fusion wearable health-monitoring system with integrated haptic feedback was previously introduced by our research group. The system's components are the following: a chest-worn device with an embedded controller board, an electrocardiogram (ECG) sensor, a temperature sensor, an accelerometer, a vibration motor, a colour-changing light-emitting diode (LED) and a push-button. This multi-sensor device makes possible to collect biometric and medical monitoring data from its wearer. The data provide a real-time indication of the wearer's health state and can also be further analysed later for medical diagnosis. The embedded vibration motor can actuate distinctive haptic feedback patterns according to the wearer's health state. The embedded colourchanging LED provides the wearer with an additional intuitive visual feedback of the current health state, and the wearer can report a potential emergency condition by using the push-button. In this paper, a conceptual case study is presented concerning possible applications for the health monitoring of elderly people in smart cities. The proposed system aims at reducing risk by assessing individual and overall potentially-harmful situations. A data collection and analysis are also presented to demonstrate that the system can provide compelling vibrotactile feedback.
\end{abstract}

Keywords—-sensor fusion, wearable, health monitoring, elderly

\section{$1 \quad$ Introduction}

Several large technology companies including Apple, Google, and Samsung are entering the expanding market of population health monitoring with the introduction of wearable devices, also known as "wearables". These devices are now at the heart of just about every discussion related to the Internet of Things (IoT) and the full range of new capabilities pervasive connectivity can bring [1] to smart cities and health monitoring applications. 


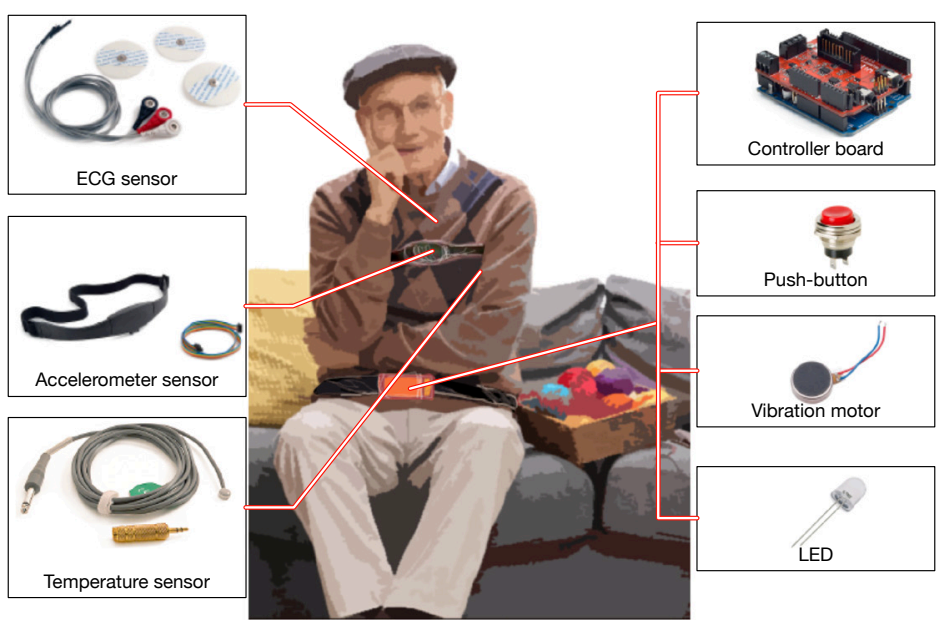

Fig. 1. The concept for a wearable multi-sensor health-monitoring system.

Wearability of smart devices is envisioned to enable novel forms of interaction, communication, cooperation and integration between humans and automated information systems. Specifically, wearables make it possible to provide haptic feedback to the human wearer during natural interaction with the environment. Haptic feedback, also known as haptics, is the use of the sense of touch in a user interface designed to supply the user with additional information. For instance, specific vibrotactile feedback may provide the user with real-time feedback about its health status.

In this perspective, the design and development of wearable health-monitoring systems (WHMS) has received lots of attention from the scientific community and industry during the last years. These low-cost systems are made up of various small sensors, transmission modules and processors. As a result, they are well-suited to wearable and unobtrusive mental and physical health status monitoring solutions and do not impose limits on time or location [2]. Even though independent sensor information is conducive to revealing the working state of the system to some extent, a comprehensive multi-parameter sensing model is more effective for analysis and evaluation of potential operational risks. To the best of our knowledge, multi-sensor fusion is one of the most suitable technologies to use when dealing with data from disparate sources [3].

Following this approach, our research group previously present a wearable health sensor monitoring system based on a multi-sensor fusion approach [4]. It consists of a chest-worn device that embeds a controller board, an electrocardiogram (ECG) sensor, a temperature sensor, an accelerometer, a vibration motor, a colour-changing light-emitting diode (LED) and a push-button. Biometric and medical monitoring applications can be performed by using this multi-sensor device. The embedded vibration motor makes it possible to actuate distinctive haptic feedback patterns according to the wearer's health state. In this way, the user can intuitively become self aware of his own health situation. The user is also provided with an additional intuitive visual feedback about the current health state by means of the embedded colour-changing 
LED. In addition, the provided push-button can be used by the user to report a potential emergency state. The proposed system opens up to a variety of application possibilities.

In this paper, we discuss the possibility of employing our system for the health monitoring of elderly people in smart cities, as shown in Figure 1. By collecting the sensor data, it is possible to monitor the health state of a user or to get sensitive data in real-time. Retrieved data can subsequently be analysed for medical diagnosis. Biometric information gathered can be wirelessly sent using the standard Wi-Fi communication protocol to a medical diagnosis center. Data can be sent to a cloud computing system to perform permanent storage or visualised in real-time by sending the information directly to a laptop or smart phone. Encryption techniques can be used to transfer data securely and to consider privacy concerns. Data mining can be performed with the raw data and could be used in conjunction with methods from the fields of artificial intelligence, machine learning, statistics and database systems by using a knowledge base. The overall goal of the data mining process is to extract information from the data set and transform it into an understandable structure so that it is possible to find previously unknown information to make inferences regarding the user's health state. In the context of constantly aging populations, maintaining persons at home represents one of the most serious challenge to be considered. The proposed system may make it possible for elderly people to live longer in their own homes.

The paper is organised as follows. A review of the related research work is given in Sect. 2. In Sect. 3, the system architecture is presented. Some ethical issues are discussed in Sect. 4. A conceptual case study describing the health monitoring of elderly people in smart cities is presented in Sect. 5. Conclusions and future work are outlined in Sect. 6.

\section{Related research works}

In the last decade, there have been numerous research efforts and products that can be classified as WHMS [2].

For example, Lv et al. [5] developed a mobile health monitoring system for the elderly. They used wireless body sensors and smart phones to monitor the wellbeing of the elderly, controlling the position, blood pressure, and ECG signal of the wearer. Rajasekaran et al. [6] presented a network that supports wearable sensors and contains on-board general computing capabilities for individual event detection, alerts, and communications with different medical services. The information is relied in real time to the PDAs of the doctor and/or caretaker. The Smart Shirt from Sensatex [7] is a wearable health-monitoring device that integrates a number of sensory devices, such as heart monitor, respiration, temperature, onto a wearable mother-board from Georgia Tech. Several other technologies have been also introduced, including the MIThril by MIT [8], e-textile from Carnegie Mellon University, and wearable e-textile from Virginia Tech [9]. Furthermore, the Lifeguard project of Stanford University is a physiological monitoring system comprising physiological sensors (ECG/respiration 
electrodes, pulse oximeter, blood pressure monitor, temperature probe), a wearable device with built-in accelerometers, and a base station. The crew physiologic observation device acquires and logs the physiological parameters measured by the sensors. More recently, Dong et al. [10] proposed an elderly health-monitoring distributed platform, which collects health data through intelligent wearable sensors and then send them to a remote server through a smartphone. Using big data technology and parallel computing, the platform uses Spark Streaming technology to carry out the online data stream processing, so as to feedback the results to the designated emergency center, hospital, or family.

Nonetheless, to the best of our knowledge, an health sensor monitoring system that features a multi-sensor fusion approach and also provides an integrated haptic feedback for the user has not yet been deeply investigated. The lack for such a system is even more evident when considering the rapidly growing needs of urban population in terms of health care. This challenge is one of the key driver for governments to increase digitization-acceleration efforts involving smart cities. The main contribution of this paper is to contribute towards the development of a wearable haptic healthmonitoring system based on sensor information fusion for the health monitoring of elderly people in smart cities.

\section{System architecture}

In this section, the proposed system architecture is presented. The framework is based on a multi-sensor fusion approach. In particular, a client-server pattern is adopted. A chest-worn device operates as a client and remotely communicates with a server where the logic of the presented architecture is implemented. In the following, the adopted design method is first described. Successively, the key elements of the system are presented. The reader is referred to Figure 2.

\subsection{Design method}

During the overall system design process, we adopted advanced virtual prototyping methods and simulations tools with the aim of improving our architectural choices, testing procedures and validation techniques. From a design point of view, virtual prototyping can be beneficial when developing complex systems. Development time can be significantly reduced, the system features can be extensively tested and quality can be assessed [11]. Therefore, virtual prototyping is necessary for validating the system design. Related simulations are carried out. In particular, this system allows for an effective risk reduction from both an individual assessment as well as an overall evaluation of the potential harm.

\subsection{Client (chest-worn device)}

The wearable device is designed to work as a client and gather different biometric data. In particular, it is a chest-worn device that consists of a controller box attached 


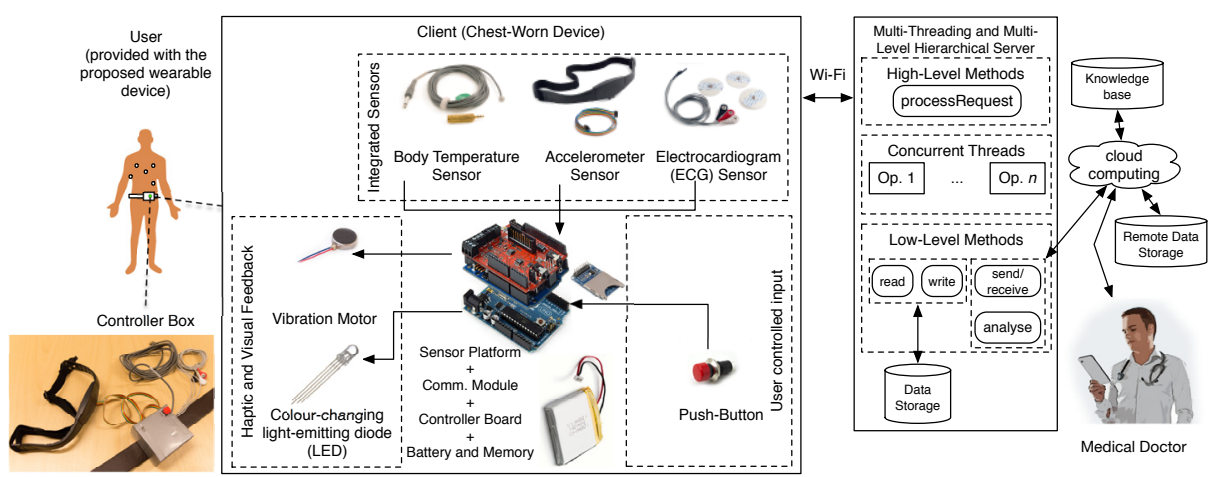

Fig. 2. The proposed system architecture.

to a belt. The device is worn by the user and it is powered by means of a polymer lithium ion battery. In the following, the components that are housed in the controller box are listed:

- Controller board: an Arduino Uno board [12] based on the ATmega328 microcontroller is used as a client. Arduino is an open-source electronics prototyping platform based on flexible, easy-to-use hardware and software. Using Arduino boards simplifies the amount of hardware and software development needed to get a system running. On the software side, Arduino provides a number of libraries to make programming the micro-controller easier. The choice of using Arduino boards makes the system framework easy to maintain and makes it possible to easily add new features in the future;

- Communication module: to give communication capabilities to the proposed wearable device, an Arduino WiFi Shield [12] is stacked on top of the adopted controller board. In detail, the Arduino WiFi Shield allows the client to communicate with the server by using the 802.11 wireless specification (WiFi). The Arduino WiFi Shield connects to an Arduino Uno board using long wire-wrap headers that extend through the shield. This keeps the pin layout intact and allows another shield to be stacked on top;

- Memory Shield Module: a Secure Digital (SD) card reader module and an SD memory card are adopted to locally store data through the Serial Peripheral Interface (SPI) by using the file system driver. This local memory is mainly used to store sensitive data such as the user's identity and other information that cannot be stored on the server side for privacy reasons. The local memory is also useful when no communication links are available. In this case, data are first temporary stored locally and then uploaded to the server whenever a connection link is available;

- Sensor platform: to gather the wearer's biometric data, an e-Health Sensor Shield [13] is stacked on top of the adopted communication module. The e-Health Sensor Shield allows Arduino boards to easily gather information from different sensors. An open-source software library is provided with the e-Health Sensor Shield, allowing for easy access to the sensor data; 
- Battery: a polymer lithium ion battery is adopted in order to provide power to the proposed controller box. This kind of battery is very slim and extremely light weight.

Different biometric sensors can be connected to the e-Health Sensor Shield according to various needs. Specifically, the following sensors are employed in our implementation:

- an ECG sensor kit, which consists of three leads (positive, negative and neutral) to be attached to the user's body. This sensor input can be used as a diagnostic tool to assess the electrical and muscular functions of the user's heart. The ECG has grown to be one of the most commonly used tests in modern medicine. In particular, this sensor has proven to be useful in the diagnosis of several cardiac pathologies ranging from myocardial ischemia and infarction to syncope and palpitations. However, the accuracy of the ECG depends on the condition being tested. A heart problem may not always show up on the ECG. Some heart conditions never produce any specific ECG changes [14];

- an accelerometer sensor, which can be placed on the user's body with a comfortable elastic band. This sensor can be used to monitor five different wearer positions (standing/sitting, supine, prone, left and right). In many cases, it is necessary to monitor the user's body positions and movements because of their connection to particular diseases (i.e., restless legs syndrome). For the particular case of elderly people, analysing movements during daily activities also helps in determining life quality and irregular pattern behaviours. The accelerometer sensor could also help to detect fainting or falling of the elderly people;

- a temperature sensor, which can be placed under the user's axilla. Body temperature is an important health state indicator and accurate measurements can provide medical insight into the wearer's response to different situations. The reason is that a number of diseases are accompanied by characteristic changes in body temperature. Likewise, the course of certain diseases can be monitored by measuring body temperature, and the efficiency of a treatment can be evaluated by the physician.

Another relevant feature of the proposed wearable device is that it provides the wearer with an intuitive haptic and visual feedback. To implement this feature, the following components are adopted:

- a vibration motor is embedded in the proposed chest-worn device. The motor is placed on the back side of the controller box and it allows for actuating distinctive haptic feedback patterns according to the wearer's health state. This feature is fundamental for improving the user's risk perception. The way in which people think, feel and behave in response to risk is receiving increasing attention, both among academics and those who are involved in promoting and regulating safety. Risk is perceived differently by different people. In this perspective, the use of haptics can significantly improve the user experience. Touch is one of the most reliable and robust senses: it is fundamental to our memory and for discerning. When the wearer's health state is normal, the motor does not produce any vibrations. If instead, 
the wearer's health state is not normal, two different vibration patterns are actuated to symbolise a potentially abnormal condition (low frequency vibrations) or a rather dangerous state of health (high frequency vibrations);

- a colour-changing LED is embedded in the proposed chest-worn device and placed on the visible side of the controller box. This LED provides the user with an additional intuitive visual feedback of the current health state. In particular, three different colours are used: green to indicate a normal health state, yellow to symbolise a potentially abnormal condition and red to point out a dangerous health state.

In addition, a push-button is embedded in the proposed wearable device, allowing the user to report a potential emergency condition or accident.

\subsection{Multi-threading and multi-level hierarchical server}

The server implements a multi-threading and multi-level control program. Strict real-time criteria are adopted. This allows to simultaneously and efficiently collect data from different users. Moreover, the chosen architectural pattern offers the following advantages: improved performance and concurrency; simplified coding of remote procedure calls and conversations; scalability and possibility to handle a growing number of users.

Three different logical levels are defined to process the client requests:

- the High-Level methods layer includes the high-level and distributed control function, processRequest, which handles each client request;

- the Concurrent Threads level is the layer where each client request is treated as a concurrent process. In detail, the health state of each user is continuously monitored by a separate thread. This level can access both the lower Low-Level methods layer as well as the higher High-Level methods layer. Each monitoring thread continuously sends the current information status back to the corresponding client so that the vibration motor and the LED can be accordingly actuated;

- the Low-Level methods layer includes the low-level functions that are used to write the acquired data to a local database (write), to read previously acquired data from a local database (read), to send the acquired data to the cloud (send), to receive previously acquired data from the cloud (receive), and to send data from the server to a knowledge base where the data can be analysed (analyse).

From an implementation point of view, the server is implemented by using the Java programming language and the corresponding standard tools for multi-threaded programming.

\subsection{Knowledge Base}

The proposed system can communicate with a knowledge base (KB). A KB may store complex structured and unstructured information collected from different sources such as public hospital databases and other health care facilities. KB technology and methods from the fields of artificial intelligence, machine learning, statistics 
and database systems can be employed to process the health measurement data and to look for unusual signs or trends.

In this initial study, the interface that provides the possibility of exposing data from the server side to an hypothetical KB was developed. In the future, rules for execution by an inference mechanism of an automation program may be implemented [15]. The rules must be based on the expertise of the health professionals. The rules would describe the range values of the health data that requires constant monitoring. In addition, the data trends (speed of increasing or decreasing values) are also important indicators that require careful monitoring by the system.

\section{$4 \quad$ Ethical issues}

To assure the user's privacy all the personal data should be protected. Data encryption must be used to transfer data securely both from the client side to the server side and from the server side to the KB. Additionally, data encryption must also be used to safely store the data.

The sensitive data flow is shown in Figure 3. All the sensitive data, such as the user's identity and location, are exclusively stored locally on the client side. Only when an emergency occurs, the client sends the user's identity and location to the server. In this circumstance, a medical alert is triggered so that medical assistance can be provided to the user. It should be noted that in this preliminary study, the user's identity and location are manually encoded on the client software. In the future, a simple graphical user interface may allow to input the user's identity while a positioning system may be integrated to track the user's location [16].

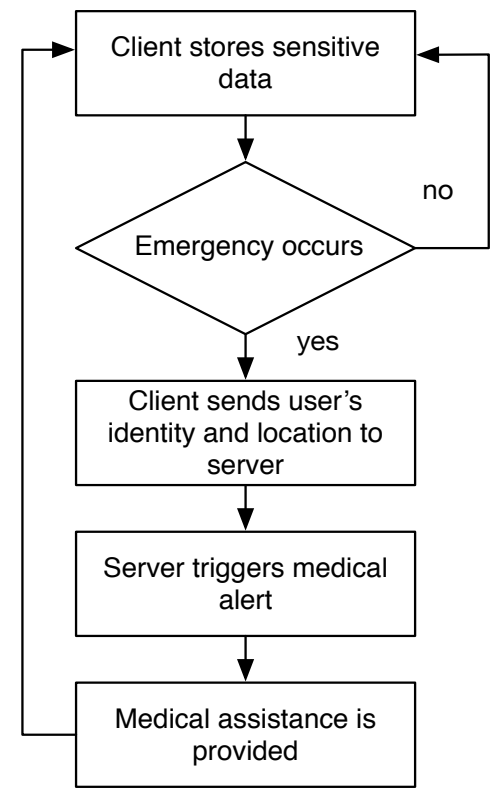

Fig. 3. Sensitive data flow 
The non-sensitive data flow is shown in Figure 4. Whenever the client collects new sensor data, the communication link availability is checked. If the communication link is not available, then the new data are temporarily stored locally. Whenever the communication link is available, data are sent from the client to the server. On the server side, the data consistency is checked against the KB. If no similar data are available on the KB, then the data are sent from the server to the KB. This process can help generate the type of robust data that is invaluable to medical researchers.

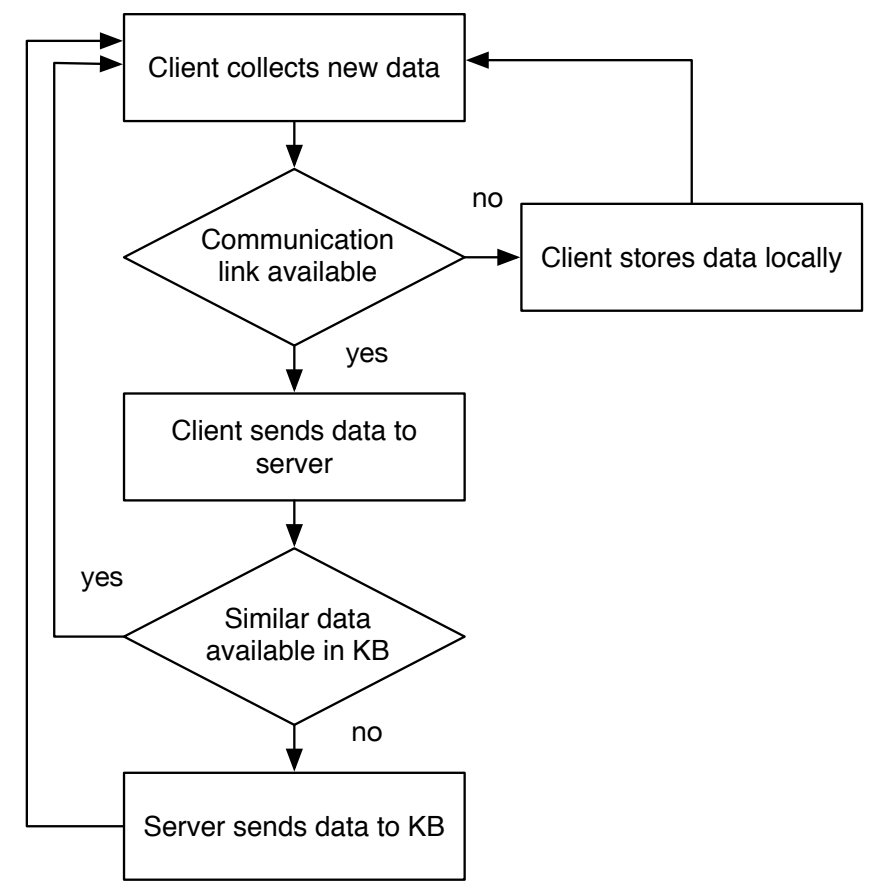

Fig. 4. Non-sensitive data flow

\section{Simulation results}

The main focus of this work is on building the system architecture and validating the communication pattern. Therefore, simulation experiments are performed with simulated data rather than real sensor data. In particular, different sample data from different open access databases are considered. Portions of these data are fed to the client to simulate real sensor measurements.

Concerning ECG monitoring, we use data from the Physiobank archive [17]. Within this archive, the MIT-BIH Arrhythmia Database [18] is considered. This database consists of 48 half-hour excerpts of two-channel ambulatory ECG recordings, obtained from 47 subjects. By using this dataset, the possibility of detecting supraventricular premature or ectopic beats (atrial or nodal) [14] is considered. While the sinoatrial node typically regulates the heartbeat during normal sinus rhythm, ectopic 
beats occur when another region of the atria depolarises before the sinoatrial node and thus triggers a premature heartbeat. Hence, a simply way to detect this event has been developed which consists of comparing the current beat time interval with the average time interval of the previous beats. If the current beat time interval is shorter than the average beat time interval, then the system detects a potential ectopic beat. For instance, this occurrence is highlighted in Figure 5, where a ECG segment plot is shown. This arrhythmia is a quite common occurrence in all ages and usually is not serious, therefore the system considers this case as a potentially abnormal condition but not as an extremely dangerous state. When this happens, the corresponding server-side monitoring thread sends a status update to the client so that the vibration motor can vibrate with low frequency and the colour of the LED can be changed to yellow.

With regard to the accelerometer sensor, the possibility of detecting five different user positions is considered. By using the software library provided with the e-Health Sensor Shield, the proposed system is able to distinguish between the following positions: standing/sitting, supine, prone, left and right. A particularly dangerous situation that can happen is fainting, which may cause the user to fall. For instance, this occurrence is highlighted in Figure 6, where the system detects a prone position for the wearer. This situation is quite dangerous and therefore the system considers this case the most critical state. When this happens, the corresponding server-side monitoring thread sends a status update to the client so that the vibration motor can vibrate with high frequency and the colour of the LED can be changed to red. A time plot of a simulated scenario is shown in Figure 7. This time plot shows the occurrences of a supine/prone position and a left/right position respectively. It should be noted that the supine and prone positions are considered equally dangerous for the user as well as the left and right positions.

Regarding the temperature monitoring, the possibility of detecting states of fever and hyperpyrexia for the user is considered. Particularly, axillary body temperature measurements are taken at 10-minutes intervals. For the sake of illustration, the case of hyperthermia is considered as an example. This occurrence is highlighted in Figure 8 , where a temperature of $38.12^{\circ} \mathrm{C}$ is measured. This is quite dangerous for the user, therefore the system considers this case as a critical state and makes the motor to vibrate with high frequency, while the colour of the LED is changed to red.

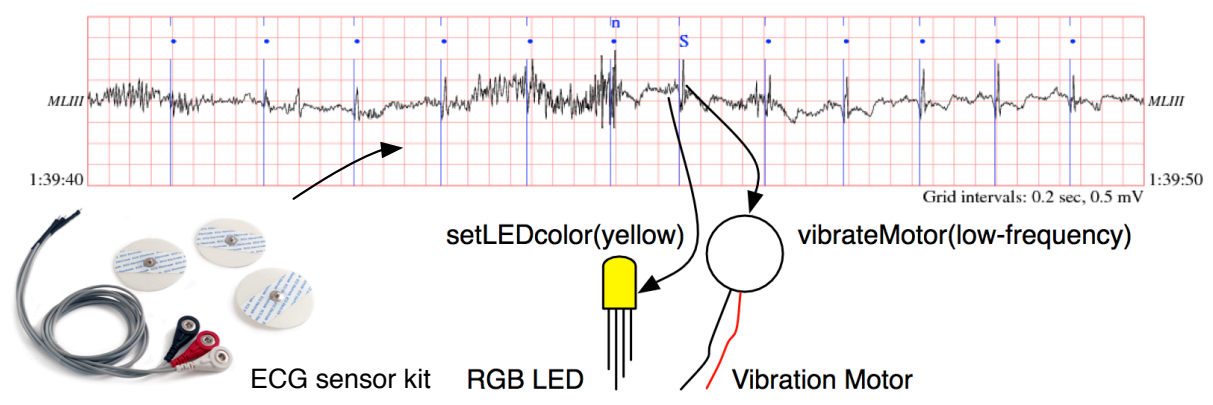

Fig. 5. An occurrence of supraventricular premature or ectopic beat, which is labeled as "S". 


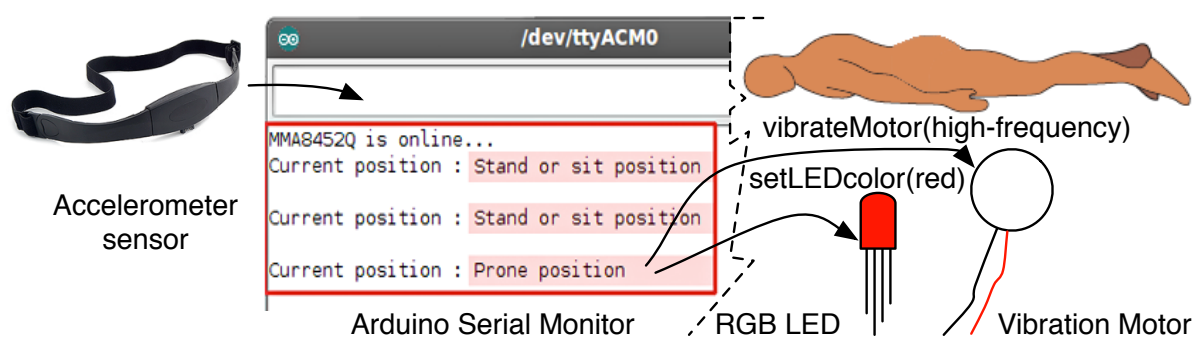

Fig. 6. An occurrence of prone position detection for the user.
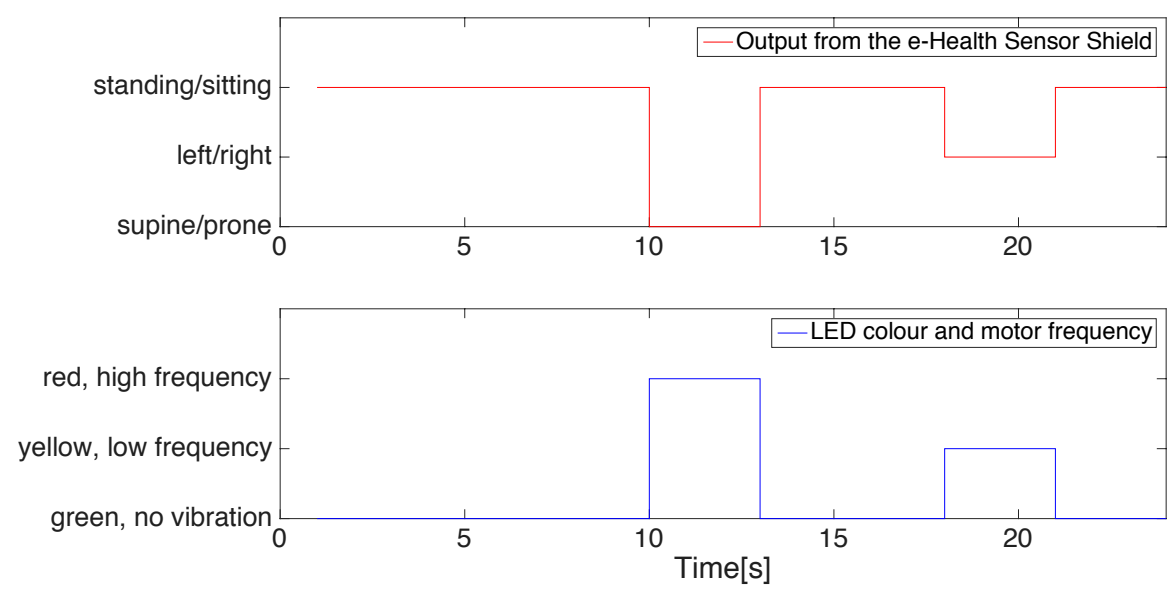

Fig. 7. A time plot of a simulated scenario showing the occurrences of a supine/prone position and a left/right position respectively.

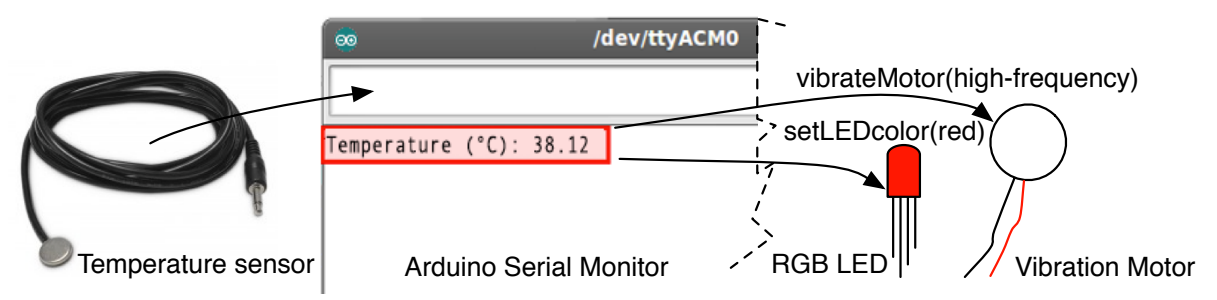

Fig. 8. An occurrence of hyperthermia is detected for the user

\section{Conceptual case study for smart cities}

A possible application scenario for the proposed system concerns the health monitoring of elderly people in smart cities. Due to a growing number of elderly people, there is a need for developing the necessary technology to make cities aware of the special needs of all their citizens including the needs of aging populations. Population 
aging is widespread across the world. This issue is most advanced in the most highly developed countries, but it is growing faster in less developed regions, which means that older persons will be increasingly concentrated in the less developed regions of the world [19]. In this perspective, there is an urgent need for developing a low-cost solution that will allow to monitor the health state and welfare of the elderly people. Such a system may make it possible for elderly people to live longer in their own homes.

The possibility of developing wearable sensor-based systems for health monitoring of elderly people has been investigated by several research groups. For instance, some usage models for health monitoring systems in daily environments based on wearable and ambient sensors were presented in [20]. An example of a possible implementation of these models was described in [15] considering the health monitoring of elderly in independent and assisted living. Nonetheless, to the best of our knowledge, a lowcost, versatile and easy to prototype system that integrates a multi-sensor fusion approach with an intuitive haptic feedback for the user has not yet been deeply investigated.

The system described in this paper meets the requirements of low-cost, versatility and rapid-prototyping. It should be noted that the production cost of the chest-worn device can easily be kept below 100 US dollars given the enclosing case, the controller boards, the communication module, the sensor platform, the sensors and the other hardware components. In the future, the production cost could be even further reduced by using a 3-D printing approach or a shape deposition manufacturing (SDM) method to produce the enclosing case. This could make the system affordable for a larger number of elderly people. The system is also versatile since the proposed multilevel architecture allows for adding new software functionalities in a modular way. The possibility of adding new sensors is guaranteed by the modularity of the proposed hardware.

A possible application scenario of the proposed system for this context is described in Figure 9. The elderly is provided with the proposed chest-worn device that allows

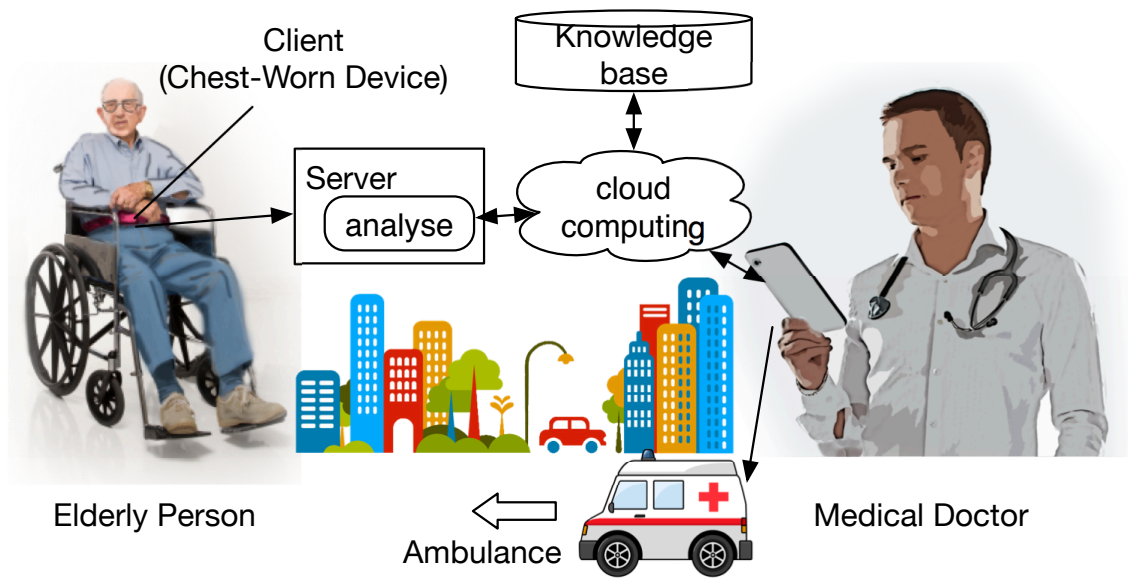

Fig. 9. An application scenario for the system concerns the health monitoring of elderly people 
for collecting biometric and medical monitoring data of its wearer. The collected data are sent from the client to the server according to the modalities described in Figure 3 and Figure 4. On the server side, the data are checked against the KB. On the KB side, the health measurement data are processed to look for unusual signs or trends. In such a case, an alarm is triggered and a notification is sent to a predefined medical diagnosis center. According to the doctor discretion, an ambulance can be sent to the elder's location so that immediate medical assistance can be provided.

\section{Conclusions and future work}

In this paper, a prototype of an integrated wearable health sensor monitoring system was presented. A multi-sensor fusion approach was adopted. The system has the following components embedded in a chest-worn device: a controller board, an accelerometer, a vibration motor, a light-emitting diode (LED) capable of changing colours, a push-button, an ECG sensor and a temperature sensor. This device makes it possible to perform biometric and medical monitoring. It is possible to actuate distinctive haptic feedback patterns in accordance with the wearer's health state thanks to the embedded vibration motor. The embedded colour changing LED provides additional intuitive feedback and the user can report a potential emergency condition by using the push-button. It is possible to use the collected biometric information in real-time for monitoring the health state of the user, or subsequently analyse the data to facilitate a medical diagnosis. The information can be sent wirelessly to a cloud computing system for permanent cloud-based data storage or it can be visualised in real time if sent to a laptop or a smart phone. In this work, the health-monitoring of elderly people was considered as a possible application scenario. Initial simulations were performed to validate the potential of the proposed system. In addition, a conceptual case study was presented to describe the possibility of employing the proposed system in the context of smart cities.

In the future, the use of experimental data will make it possible to certify system performance in a real application scenario. To do this, an extensive field test is necessary involving a large number of users. A comprehensive experimental test would also make it possible to assess the proposed system from a human factor point of view. Another challenge that could be explored in the future is the use of advanced techniques for data mining that draw from the fields of big data, artificial intelligence, machine learning, statistics and database systems by using a knowledge base. Optimisation methods and software libraries similar to the ones proposed in [21] may be adopted.

In the near future, we expect wearable technology to deliver a key interface and input into the industrial Internet of Things (IoT). The proposed system contributes towards the fulfillment of this idea. 


\section{References}

[1] M. Swan, "Sensor mania! the internet of things, wearable computing, objective metrics, and the quantified self 2.0," Journal of Sensor and Actuator Networks, vol. 1, no. 3, pp. 217-253, (2012). https://doi.org/10.3390/jsan1030217

[2] A. Pantelopoulos and N. G. Bourbakis, "A survey on wearable sensor- based systems for health monitoring and prognosis," IEEE Transactions on Systems, Man, and Cybernetics, Part C (Applications and Reviews), vol. 40, no. 1, pp. 1-12, (2010). https://doi.org/10.1109/TSMCC.2009.2032660

[3] G.-Z.Yang,J.Andreu-Perez,X.Hu,andS.Thiemjarus,"Multi-sensor fusion," in Body sensor networks. Springer, (2014), pp. 301-354.

[4] F. Sanfilippo and K. Y. Pettersen, "A sensor fusion wearable health-monitoring system with haptic feedback," in Proc. of the 11th IEEE International Conference on Innovations in Information Technology (IIT15), Dubai, United Arab Emirates. IEEE, (2015), pp. 262266. https://doi.org/10.1109/INNOVATIONS.2015.7381551

[5] Z. Lv, F. Xia, G. Wu, L. Yao, and Z. Chen, "icare: a mobile health monitoring system for the elderly," in Proc. of the 2010 IEEE/ACM International Conference on Green Computing and Communications \& International Conference on Cyber, Physical and Social Computing, (2010), pp. 699-705. https://doi.org/10.1109/GreenCom-CPSCom.2010.84

[6] M. P. Rajasekaran, S. Radhakrishnan, and P. Subbaraj,"Elderlypatient monitoring system using a wireless sensor network," Telemedicine and e-Health, vol. 15, no. 1, pp. 73-79, (2009). https://doi.org/10.1089/tmj.2008.0056

[7] S. Park, K. Mackenzie, and S. Jayaraman,"Thewearablemotherboard: a framework for personalized mobile information processing (pmip)," in Proc. of the 39th annual design automation conference, (2002), pp. 170-174.

[8] T. Martin, M. T. Jones, J. Edmison, and R. Shenoy,"Towardsadesign framework for wearable electronic textiles." in ISWC, vol. 3, (2003), pp. 190-200.

[9] D. Marculescu, R. Marculescu, and P. K. Khosla, "Challenges and opportunities in electronic textiles modeling and optimization," in Proc. of the 39th annual design automation conference (ACM), (2002), pp. 175-180.

[10] M. Dong, X. Huang, S. Bi, X. Zeng, N. Pang, H. Liu, and X. Tang, "The elderly health monitoring platform based on spark," in Proc. of the 2015 IEEE International Conference on Cyber Technology in Automation, Control, and Intelligent Systems (CYBER), (2015), pp. 514-519. https://doi.org/10.1109/CYBER.2015.7287992

[11] G. G. Wang, "Definition and review of virtual prototyping," Journal of Computing and Information Science in engineering, vol. 2, no. 3, pp. 232-236, (2002). https://doi.org/10.1115/1.1526508

[12] Arduino. (2017, January) Arduino, an open-source electronics prototyping platform. [Online]. Available: http://arduino.cc/.

[13] Cooking Hacks. (2017, January) e-Health Sensor Shield. [Online]. Available: http://www.cooking-hacks.com/.

[14] J. McNames, M. Aboy, B. Goldstein et al., "Prediction of paroxysmal atrial fibrillation by analysis of atrial premature complexes," IEEE Transactions on Biomedical Engineering, vol. 51, no. 4, pp. 561-569, (2004). https://doi.org/10.1109/TBME.2003.821030

[15] G. K. Pang, "Health monitoring of elderly in independent and assisted living," in Proc. of the IEEE International Conference on Biomedical Engineering (ICoBE), (2012), pp. 553556. https://doi.org/10.1109/ICoBE.2012.6178978 
[16] F. Sanfilippo and K. Y. Pettersen, "XBee positioning system with embedded haptic feedback for dangerous offshore operations: a preliminary study," in Proc. of the MTS/IEEE Oceans '15 Conference, Genova, Italy, (2015), pp. 1-6.

[17] G. B. Moody, R. G. Mark, and A. L. Goldberger, "Physionet: a web- based resource for the study of physiologic signals," IEEE Engineering in Medicine and Biology Magazine, vol. 20, no. 3, pp. 70-75, (2001). https://doi.org/10.1109/51.932728

[18] A. L. Goldberger, L. A. Amaral, L. Glass, J. M. Hausdorff, P. C. Ivanov, R. G. Mark, J. E. Mietus, G. B. Moody, C.-K. Peng, and H. E. Stanley, "Physiobank, physiotoolkit, and physionet components of a new research resource for complex physiologic signals," Circulation, vol. 101, no. 23, pp. e215-e220, (2000). https://doi.org/10.1161/01.CIR.101.23.e215

[19] W. Lutz, W. Sanderson, and S. Scherbov, "The coming acceleration of global population ageing," Nature, vol. 451, no. 7179, pp. 716-719, (2008). https://doi.org/10.1038/nat ure06516

[20] I. Korhonen, J. Parkka, and M. Van Gils, "Health monitoring in the home of the future," Engineering in Medicine and Biology Magazine, IEEE, vol. 22, no. 3, pp. 66-73, (2003). https://doi.org/10.1109/MEMB.2003.1213628

[21] L. I. Hatledal, F. Sanfilippo, and H. Zhang, "JIOP: a java intelligent optimisation and machine learning framework," in Proc. of the 28th European Conference on Modelling and Simulation (ECMS), Brescia, Italy, (2014), pp. 101-107.

\section{Authors}

Filippo Sanfilippo is a member of the Cyber Physical and Applied Smart Systems Research Group, Dept. of Science and Industry systems, Faculty of Technology, Natural Sciences and Maritime Sciences, University College of Southeast Norway (USN), 3603 Kongsberg, Norway. Email: filippo.sanfilippo@usn.no.

Claudio Pacchierotti is a member of the CNRS, University of Rennes, Inria, IRISA, 35000 Rennes, France. Email: claudio.pacchierotti@irisa.fr.

Article submitted 14 March 2018. Final acceptance 22 April 2018. Final version published as submitted by the authors. 